\title{
Entrevista a Sergio Larraín García-Moreno: Pionero de la modernidad en Chile ${ }^{* * *}$
}

\author{
Interview with Sergio Larraín García-Moreno
}

<Resumen>

Entrevista inédita realizada por los arquitectos Humberto Eliash y Teresa Lima-Campos, al arquitecto y Premio Nacional de Arquitectura Sergio Larraín García-Moreno. En la conversación se revisará parte de su formación en la Pontificia Universidad Católica, el temprano contacto con las vanguardias artísticas y arquitectónicas europeas de la primera mitad del siglo xx y sus opiniones sobre la arquitectura moderna en Chile, de la que es considerado Fundador y arquitecto fundamental.

$<$ Abstract $>$

An unpublished interview with the architect and national award Sergio Larrain Garcia Moreno, conducted by the architects Humberto Eliash and Teresa Lima-Campos. In the conversation they will revise part of his education at the Pontificia Universidad Católica, the early contact with the avant-garde European art and architecture of the first half of the twentieth century and his views on modern architecture in chile, of which he is regarded as a founder and a fundamental architect.

<PALABRAS CLAVE>

ARQUI TECTURA CHILENA / MOVIMIENTO MODERNO / ENSEÑANZA DE LA ARQUITECTURA

\section{<KEYWORDS >}

CHILEAN ARCHITECTURE / MODERN MOVEMENT / TEACHING ARCHITECTURE

\section{Introducción}

Don Sergio Larraín es una de las figuras más relevantes de la arquitectura chilena del siglo xx. Nació en Chile en el año 1905 y realizó sus estudios secundarios en Chile, Suiza y Francia. Estudió arquitectura en la P. Universidad Católica de Santiago, titulándose en el año 1928, con distinción máxima. Posteriormente realizó estudios complementarios en Chile y en el extranjero, con una breve pasada por la Bauhaus en los tiempos en que la dirigía Hannes Meyer ${ }^{1}$

Dentro de su actividad universitaria, cabe destacar su labor como profesor de «Historia del arte» y «Taller», y su labor como Decano en la Facultad de Arquitectura y Bellas Artes de la P. Universidad Católica de Chile (1953 y 1956). En el año 1953 funda la Escuela de Arte y Diseño, trayendo a Josef Albers $^{2}$ y a otros profesores de universidades norteamericanas, que ejercieron una gran influencia en las generaciones de arquitectos formados durante esos años. En el año 1965 funda el Centro Interdisciplinario de Desarrollo Urbano y Regional, CIDU, con el apoyo de la Fundación Ford.

Sergio Larraín García-Moreno desarrolló una dilatada y constante labor profesional, desde el año 1929, año en que proyecta y

\footnotetext{
* Arquitecto Titulado en la Facultad de Arquitectura y Urbanismo de la Universidad de Chile. Ha ejercido la profesión de manera independiente o asociada, desarrollando una importante producción de obras, dictando conferencias en diferentes países. Ha sido profesor de la Escuela de Arquitectura de la Universidad de Chile y profesor invitado de distintas universidades Latinoamericanas.

*** Arquitecta PUC.

Arquitecto suizo que fue el segundo director de la Bauhaus, entre 1928 y 1930

2 Josef Albers (1888-1976). Artista y profesor alemán, creador de algunos de los programas de educación más importantes e influyentes del siglo xx en el ámbito artístico. Fue profesor de la Bauhaus y emigró a EE.UU., luego de la llegada de los nazis al poder, ejerciendo como académico en Black Mountain College, Carolina del Norte y en la Universidad de Yale, donde fue director del departamento de Diseño.
} 
construye el edificio Oberpaur ${ }^{3}$ considerado por muchos el primer edificio de arquitectura moderna en Chile, realizando numerosos edificios públicos y privados, de vivienda y de equipamiento. Aparte de los trabajos realizados en combinación con otros arquitectos, en Chile y en el extranjero, se destaca su asociación con Jorge Arteaga (1929-1938), con Emilio Duhart (1940-1960), y su asociación con los arquitectos Jorge Swinburn e Ignacio Covarrubias a partir del año 1960.

En 1972, recibió la Medalla de Oro, premio de honor del Colegio de Arquitectos de Chile, por su destacada labor profesional. Entre sus actividades extraprofesionales, cabe destacar su labor como regidor por Santiago (19361940). Su participación en la $2^{\text {da }}$ Guerra como agente antinazi colaborando con el Gobierno Británico desde Chile, su labor como Embajador de Chile en el Perú (1960-1970), y la creación del Museo de Arte Precolombino en Santiago, con la donación que hiciera de su colección de piezas precolombinas que se considera entre las más importantes de Latinoamérica.

El siguiente es el texto de la entrevista realizada en su casa de Pedro de Valdivia Norte, el 28 de abril de 1982, por los arquitectos Teresa de Lima Campos y Humberto Eliash.

\section{Entrevista}

Don Sergio, cómo fue su educación secundaria en Europa, y cómo se gestó su interés por la arquitectura, y más propiamente por la arquitectura moderna, que por esos años hacia su aparición en las vanguardias europeas. Recordemos que los años 20 fueron muy importantes para la definición del arte y la arquitectura moderna.

-Bueno me fui a Europa creo que en $2^{\circ}$ ó $3^{\circ}$ año de humanidades ${ }^{4}$, fue en el año 1920. Se enfermó una hermana, que no se podía curar aquí, y nos fuimos a Europa. La verdad es que yo estuve muy solo en Europa, no tenía amigos. Estuve en un colegio en Suiza, después estuve en un colegio en Francia después tenía profesores privados, después me aburrían los regímenes escolares y pedía profesores de acuerdo con mis gustos, también cambié de padres.
Dentro de esta soledad de amigos, de gente de mi edad, -por curiosidad se aprenden las cosas (...)- me fui interesando por la cuestión artística más y más. Naturalmente era un momento extraordinariamente interesante en todo el arte: en la pintura, en la música, en la poesía. Me interesé mucho durante ese tiempo por las cuestiones de arte, por el movimiento de arte, de pintura, de poesía. Además me interesé por el arte en general. Lo que más me fascinó fue todo el arte medieval. Me encantaban las catedrales, las iglesias medievales, en particular los conventos románicos, y las iglesias góticas, me fascinaban. Tanto que con los dineros que economizaba, cuando tenía 16 ó 17 años, compraba objetos que tengo todavía aquí en mi casa, hoy día de gran valor. Tengo un cofre gótico, por ejemplo, tengo una virgen burguiñona ${ }^{5}$ del siglo xIv que la compré toda rota y que me costó 90 francos, y que es preciosa, realmente una escultura preciosa. Cosas románicas compré también, que las encontraba en cachureos y que valían muy poco en ese tiempo. Me fui interesando más y más en ese mundo. No tenía la oportunidad para tener correrías nocturnas o cosas de vida más o menos libertina, porque me dejaban muy poca libertad en la casa.

\section{¿Ud. terminó la educación secundaria allá?}

-Terminé una educación secundaria más o menos relativa, porque no sé si es educación la que yo tuve. Terminé mis estudios, di una primera parte del bachillerato en Francia, no di la segunda. Me vine aquí a Chile, pero en realidad yo tenía una cultura muy desordenada, en general de un nivel más alto que el que tenían los muchachos de mi edad aquí, y con mucha más apertura hacia una cantidad de cosas que estaban ocurriendo y que aquí nadie sabía. Por lo menos entre la gente de mi edad en esa época.

¿Era mucho más difícil la comunicación en esa época? ¿Era mucho más "distante» la relación entre la cultura Europea y Latinoamericana?

-Yo creo que seguimos viviendo todavía bastante desplazados, y desfasados en el tiempo. Son pocos los que prestan atención a los movimientos que se están gestando, pero
Sergio Larraín García-Moreno.

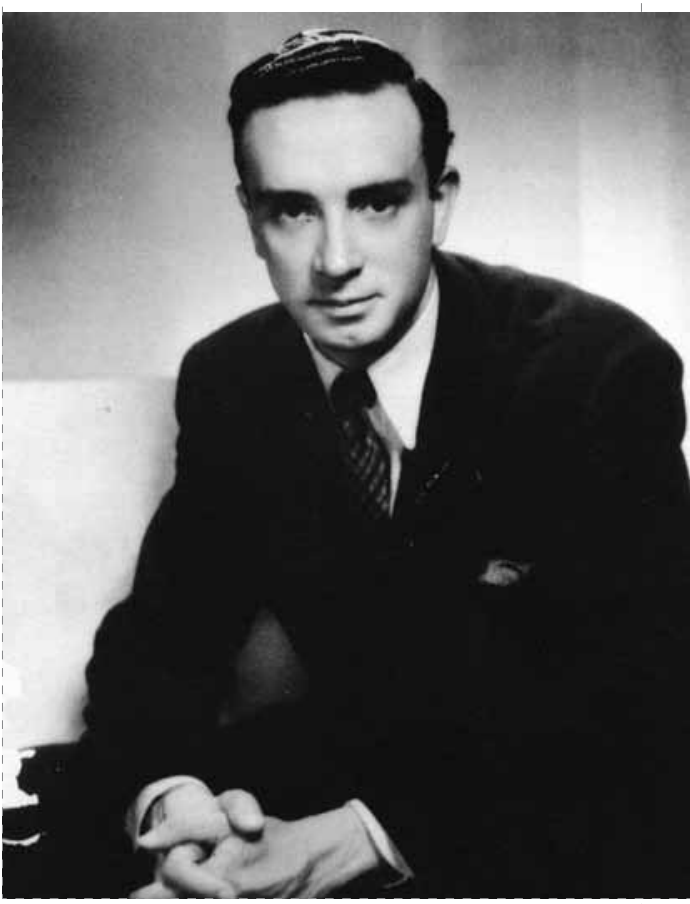

no se dan en el ambiente. Yo creo que si te vas a las escuelas de arquitectura hoy día, en los primeros años los alumnos no saben verdaderamente cual es el panorama de hoy día, no saben qué es la arquitectura de hoy, ni qué es la arquitectura, ni lo que están estudiando.

Dentro de todo, estaba Vicente Huidobro ${ }^{6}$ allá en Francia, que era muy amigo de un hermano. A través de él me tocó conocer a Vicente, y conocer un poco el movimiento de poesía, el dadaísmo, y a Apollinaire ${ }^{7}$, que había muerto, pero conocí su obra. Estaba viviendo entre todo este deambular por e mundo del arte antiguo y moderno, de ir a conciertos, de ir a exposiciones, de leer revistas de arte, de oír discusiones, los ánimos estaban muy exaltados. Yo en la medida que podía hacer cosas, y me podía meter, me metía, pero evidentemente yo no pertenecía ni tenía una edad para participar en forma activa. Yo era un extranjero, no pertenecía a ningún grupo, no practicaba ningún arte, estaba estudiando desordenadamente y leyendo mucho, interesándome por muchas cosas. Mi hermano, como digo, que era

El edificio Oberpaur de los arquitectos Arqtos. S. Larraín G-M. y J. Arteaga. ubicado en la esquina sur-oriente de las calles Huérfanos y Estado, en Santiago.

$8^{\circ}$ ó $9^{\circ}$ año de formación escolar, de un total de 12 divididos en primaria (6 años) y humanidades ( 6 años).

De los pueblos originarios de la hoy Borgoña, Francia.

(1893-1948) Poeta chileno, vinculado a la corriente creacionista de la poesía chilena. Durante la década del 20 colabora en París, con Amedeé Ozenfant y Le Corbusier en

L'Esprit Nouveau.

Guillaume Apollinaire (1880-1918), escritor francés. Inventor del término surrealismo y surrealista. 
muy amigo de Vicente Huidobro, estaba muy metido en todas estas cosas. Me hacia comprar revistas como L' Espirit Noveau ${ }^{8}$, por ejemplo, todavía tango algunos números más o menos de esos tiempos. Mi hermano me regaló un libro de Le Corbusier que se llamaba «Vers Une Architecture»9. Tengo la edición original todavía, junto con todas las obras de Le Corbusier, que están en mi oficina. Yo leí ese libro, y tengo el recuerdo muy claro de que la arquitectura que él proponía la hallaba bastante repulsiva, en el sentido de que la hallaba inhumana. Es decir, al lado de lo cálida y de lo atrayente que resultaba la arquitectura, por ejemplo, medieval; esta arquitectura de Le Corbusier era como una arquitectura esquemática, una arquitectura de contornos y que perdía interés cuando uno se acercaba a las cosas. Porque las murallas no vivían, en cambio las murallas de una iglesia gótica o románica, con el tratamiento que tenían, la textura (palabras todas que aprendí mucho después naturalmente), me hablaban. Tenían un interés mucho más grande, era como ver una flor, era como ver una cosa viva, se podía mirar los detalles.

Por ejemplo, a la Catedral de Nuestra Señora de Chartres uno la mira en el paisaje desde lejos y es una maravilla, está insertada perfectamente en la «Plaine de Bon», es perfecta. Después en el pueblo de Chartres, es perfecta, en la plaza es perfecta, uno se mete al pórtico y empieza luego a mirar los detalles, a mirar la piedra, a mirar las esculturas, luego entra a mirar los vitrales, la luz, todo, todo es como un resplandor, es verdaderamente un milagro. En cambio en estas casas era como meterse dentro de un teorema de Euclides, uno miraba y decía: bueno en realidad está todo en orden, está todo bien hecho, pero no me conmovía verdaderamente. No encontraba en eso poesía. Sin embargo, me fue atrapando más y más. Me interesó el aspecto más bien ético de la Arquitectura que se estaba haciendo. Entonces verdaderamente parecía que Le Corbusier estaba reaccionando ante una realidad que era la realidad de su época, en que todo el mundo estaba mintiendo.
Se estaba haciendo una arquitectura de una fastuosidad que no correspondía ni a la verdad de los materiales, ni a la verdad de la estructura, ni a la verdad de las funciones, ni a la verdad urbana, ni a nada. Parecía que era necesario hacer un movimiento de depuración moral, ética más que estética.

A pesar de la resistencia que yo encontraba en mí mismo, me fui interesando cada vez más. En ese momento pensé: voy a ser arquitecto, es lo único que puedo hacer. Yo no tengo ninguna dote muy particular para ningún arte. No soy ni músico, ni escritor, ni poeta, ni muy buen dibujante, ni ninguna de esas cosas. Pero lo que me parecía que podía estar dentro de mis posibilidades era la arquitectura. Tomé la decisión de estudiar arquitectura cuando llegara a Chile y me vine en el año 1924 Conseguí entrar a la Escuela de Arquitectura sin el bachillerato requerido, con un permiso especial que me dio don Crecente Errázuriz, Arzobispo de Santiago, a quien le conté toda mi historia y estuvo conversando largo conmigo. Era un caballero muy ilustrado y muy inteligente y me dijo: «Hijo, tú tienes el nivel para poder entrar a la Escuela, así que no te preocupes, yo te libero». Entonces entré a la Escuela sin el bachillerato chileno que era obligatorio en ese momento.

Yo creo que mi entrada a la Escuela me puso muy vanidoso, porque yo sentí que ya estaba en otro nivel cultural, respecto de todo el curso. Yo sabía cosas que nadie había oído, y no sólo sabía cosas que nadie había oído en mi curso, sino que ningún profesor había oído. Cosas que ningún arquitecto chileno había oído. No sabían nada de nada, no conocían n los orígenes del movimiento. De manera que yo me empecé a sentir bastante pretencioso. Empezaba a dar conferencias, a contar y a explicar las cosas. Recuerdo que a don José Forteza ${ }^{10}$, que era profesor, le presté todas las obras de Le Corbusier.

Yo les decía a los profesores: «lo que ustedes nos enseñan, ya no existe, esto ya no es arquitectura», y respondían: «¿cómo entonces se enseñó en el Beaux Arts?», y yo les contestaba: «pero si nadie cree en el Beaux Arts, ningún arquitecto cree, ningún artista cree en el Beaux Arts». La única persona que me oyó fue don José Forteza, me pidió que le prestara mis libros. Entonces le presté los libros y me los devolvió desgraciadamente empastados con una pasta bien horrorosa, les sacó la tapa y así están ahora. Pero aprecio ese gesto de don José, a él esto le parecía muy interesante. En ese tiempo yo recuerdo que hubo un concurso de la Sociedad de las Naciones, mientras yo aún estaba en la universidad, un concurso de un edificio que se iba hacer en Ginebra, de un edifico muy importante, de la Societé des Nations ${ }^{11}$. Le Corbusier propuso un proyecto que era muy revolucionario, verdaderamente interesante, que a lo mejor hoy día no nos gustaría, pero en ese momento era verdaderamente como un grito.

Y es el proyecto que pasó a la historia, porque del otro nadie se recuerda.

-El otro no tiene el menor interés, yo lo he visto construido. Le Corbusier escribió un libro que se llamaba "Une maison, Un palais» ${ }^{12}, \mathrm{y}$ hubo una polémica bastante grande. En ese entonces me suscribí en Francia a revistas de todas partes. Durante mi estada en la universidad, recibía revistas como «De Stij 13» de Holanda, «Moderne Bauformen» ${ }^{14}$, o "L'Architecture Vivante» 15. También estaba suscrito a los "Cahiers d' Art» ${ }^{16}$, tengo la colección completa desde el primer número hasta el último.

Mi vida ha correspondido a toda esta generación del Arte Moderno. Picasso comenzó a pintar en el año 1900, 1902, pero verdaderamente el impacto del Arte Moderno fue después de la primera Guerra Mundial. El período en que yo me incorporé, en que me asomé a la ventana de todas estas cosas, fue por ahí por el año 20. Recuerdo que los primeros proyectos que hice, fueron proyectos que yo pensaba que eran de arquitectura

8 Le Corbusier junto con Paul Dermée y Amedeé Ozenfant fundan la revista L' Esprit Nouveau (El Espíritu Nuevo), publicación que permite divulgar sus pensamientos sobre el arte y la arquitectura y que alcanzara una importante repercusión internacional.

- Vers une Architecture (Hacía una arquitectura) publicado originalmente en el año 1923 en París.

10 José Forteza Ubach, Arquitecto Catalán residente en Chile. En 1889 es declarado Arquitecto de Obras Públicas durante el gobierno de Balmaceda. Desarrolló una destacada carrera docente en las Universidades de Chile y Católica. Autor del Hotel Bristol, Monumento Nacional desde 2007 y del desaparecido Palacio Undurraga, entre numerosas obras.

El primer lugar del concurso fue otorgado al proyecto de los arquitectos Henri-Paul Nénot y Julien Flegenheimer,

12 Publicado originalmente en el año 1928 por Le Corbusier.

13 Revista dedicada a temas relativos a las artes plásticas, fundada por Theo Van Doesburg y Piet Mondrian y desarrollada entre los años 1917 y 1931.

14 Publicación alemana asociada a la Bauhaus, de alta influencia durante la primera mitad del siglo xx.

15 Revista Francesa editada por Albert Morancé y Jean Badovici. Se publicó entre los años 1923 y 1933, editando un total de 42 números.

16 Revista Francesa dedicada al arte, fundada por el galerista Christian Zervos, en la que participaron algunas de las figuras más importantes del mundo artístico de la primera mitad del siglo $x x$. 
Moderna, que en realidad eran bastante copiados, o muy influenciados, no tenían ni la capacidad ni la creatividad, ni tampoco el conocimiento suficientemente profundo de las raíces de estos movimientos, de manera que uno más bien se vinculaba con las formas y con la expresión. Creo que el primer proyecto que hice fue un mercado, yo estaba en $2^{\circ}$ año de Arquitectura. Recuerdo que de él me dijeron: "este proyecto no se puede ni examinar»(comenta el arquitecto entre risas).

Todos nos enseñaban las órdenes. Se partía estudiando el Vignola ${ }^{17}$, y nos criticaban si las proporciones no estaban correctas, nos median con compás de punta seca, para ver si la proporción era tal o cual, nos criticaban si las sombras estaban mal hechas, todo eso era muy importante. Era una cosa completamente estúpida. Vignola, por supuesto era un gran arquitecto, después lo he venido a descubrir En ese momento yo lo odiaba. Pero después me di cuenta que Vignola en su tiempo fue un arquitecto que nunca pretendió que su arquitectura fuera un dogma para todas las generaciones, sino que era la arquitectura de su época, y la facilitó en un manual para el uso de su época como lo que había pasado con Vitruvio ${ }^{18}$, y como pasó con muchos arquitectos de los siglos XVII y XVIII.

Me parece que el ambiente que se vivía en la Escuela de Arquitectura aquí era un poco acrítico en general, de parte de profesores $y$ estudiantes.

-Enteramente acrítico. Había un profesor que nos hacía una clase que se llamaba «Teoría de la Arquitectura», se hacía con un libro francés que se llamaba el Guadet ${ }^{19}$. Yo sabía francés, de manera que me transformé en el traductor oficial del curso. Entonces leía en español y los alumnos tomaban apuntes, de manera que además yo estaba muy informado de la arquitectura en la enseñanza oficial, estaba informado del otro lado ¿me entiendes? Yo tenía en el curso realmente menos condiciones de arquitecto que muchos de mis compañeros, pero en cambio tenía información y estaba metido en un nivel intelectual y artístico que eran enteramente incomparables con todos ellos.

\section{¿Y durante toda su permanencia en la Escuela, se desarrolló esta pugna entre las cosas que usted conocía y el tipo de enseñanza que le estaban imponiendo?}

-Así es, de tal manera que yo a veces cedía. Yo no sé si les conté una anécdota que me pasó con un profesor que se llamaba Gustavo García Postigo, que es el autor de la Biblioteca Nacional ${ }^{20}$, edificio que también yo odiaba y me parecía un vómito (risas). Uno era muy intransigente en esa época, y muy dogmático. Entonces todos esos adornos, esas cosas, todos esos colgajos que tenía, la cantidad de columnas, la ubicación en la Alameda, todo me parecía un horror. Él fue nuestro profesor y nos dio como tema un Club Militar. Me dijo a mí: «Yo le prevengo jovencito Larraín, usted no me va venir con sus historias de arquitectura moderna aquí, usted va hacer la verdadera arquitectura, usted va hacer arquitectura aquí, no esas cosas de construcción. Usted va hacer un club militar con órdenes perfectos». Entonces yo tomé de una revista americana a la que estaba suscrito, la «Architectural Record ${ }^{21}$, unos levantamientos que había de arquitectura renacentista y tome el Campidoglio de Roma ${ }^{22}$, de Miguel Ángel y lo copié, luego hice la planta para que coincidiera con el club. Era un club militar con un hall redondo, que se parecía al Royal Automóvil Club de Londres ${ }^{23}$, un edificio interesante para su época, y todo coincidía. Salió de lo más bien, cupo perfectamente dentro del Campidoglio fíjate, (risas) se desarrolló perfectamente. Yo llegué con mi proyecto y la comisión que dirigía don Gustavo me dijo «¿Qué son estas proporciones? ¡Este no es clásico ni es nada!», y empezó a medir con compás. Me dijo «pero si esto no es Vignola no es nada, esto le pasa por la ignorancia en que usted ha vivido por hacer puras cosas modernas». No me atrevía a decirle en el examen «Señor, si este es de Miguel Ánge|». Finalmente se lo dije: «Don Gustavo, aquí tengo el original, éste es el Campidoglio de Miguel Ángel». Entonces lo miró y me dijo: «Ah, si esta es una revista americana ¡quién sabe qué levantamientos son estos que hacen los americanos, si los americanos no saben arquitectura!». Entonces me anuló también la fuente, porque no se atrevió a hablar contra Miguel Ángel. En fin, ese era el ambiente que había.

Entre sus compañeros, entre los propios estudiantes, justed recuerda al alguno que haya asumido esta posición más crítica?

-Claro, hubo alguno. Desde luego estuvo Andrés Garáfulic ${ }^{24}$. Nos recibimos el mismo día, pero curiosamente, él se recibió con una catedral gótica, era un proyecto muy bonito. Él sí que tenía una mano de dibujante que yo no tenía, un dibujo extraordinario, pero juna catedral gótica! Yo me recibí con mi Estación de Ferrocarril y nos examinaron en la misma tarde y nos pusieron a los dos una nota óptima. Eran dos proyectos verdaderamente muy completos, muy buenos, muy serios los dos. Andrés Garáfulic, en el curso, hizo algunos proyectos de arquitectura moderna. Pero eran como incursiones. Él tenía bastante libertad y bastante creatividad, era cercano al expresionismo.

Me acuerdo que cuando llegué de Europa yo le propuse que nos asociáramos y me dijo «no puedo porque ya me acabo de asociar con Costábal» ${ }^{25}$. Entonces me invitó a asociarme Jorge Arteaga ${ }^{26}$, una persona que había sido amiga de mi familia, un arquitecto clásico, sin embargo las cosas que él hacia a mí me parecían muy respetables.

Arteaga me enseñó realmente el oficio de arquitecto. Él tenía una oficina muy bien montada, se trabajaba muy bien y aprendí a ser arquitecto, yo era un jovencito un poco pedante cuando me recibí, pero realmente no sabía nada. Yo iba a las obras, por ejemplo, y delante de los estucadores, de los albañiles y de todo esto, no me atrevía a corregirlos porque ya veía que ellos sabían lo que yo no sabía y creo que sigue pasando todavía en las escuelas de arquitectura. En cambio, con Arteaga que tenía mucho oficio y que sabía mucha construcción, yo me fui atreviendo a

Tratado de las cinco órdenes de la Arquitectura escrito en al año de 1562 por el arquitecto italiano Giacomo Barozzi de Vignola.

18 Marco Vitruvio Polión, arquitecto romano del siglo i a.C. Es autor del tratado De Arquitectura, constituido por 10 libros, el tratado más antiguo que se conserva de arquitectura.

Arquitecto francés, autor de Elements et Theorie de l'Architecture.

García Postigo ganó ese concurso en 1913.

Architectural Record es una revista mensual editada en Estados Unidos, convertida en la actualidad en una de las revistas de arquitectura más importantes del mundo.

22 Proyecto diseñado por Miguel Ángel que se lleva a cabo durante la década de 1550 y que es terminado en el siglo xvil.

23 The Royal Automobile Club, London. Diseñado por los arquitectos Arthur Joseph Davis y Charles Mewes. Construido entre los años 1908 y 1911.

24 Andrés Garáfulic Yankovic. 1905-1956. (Arquitecto). Desarrolló algunas de las primeras obras de arquitectura moderna en Chile. Autor de la basílica de la Virgen de Lourdes en Santiago.

Eduardo Costábal, arquitecto Pontificia Universidad Católica de Chile, que desarrolló una exitosa dupla con Andrés Garáfulic.

26 Jorge Arteaga, arquitecto Pontificia Universidad Católica de Chile 

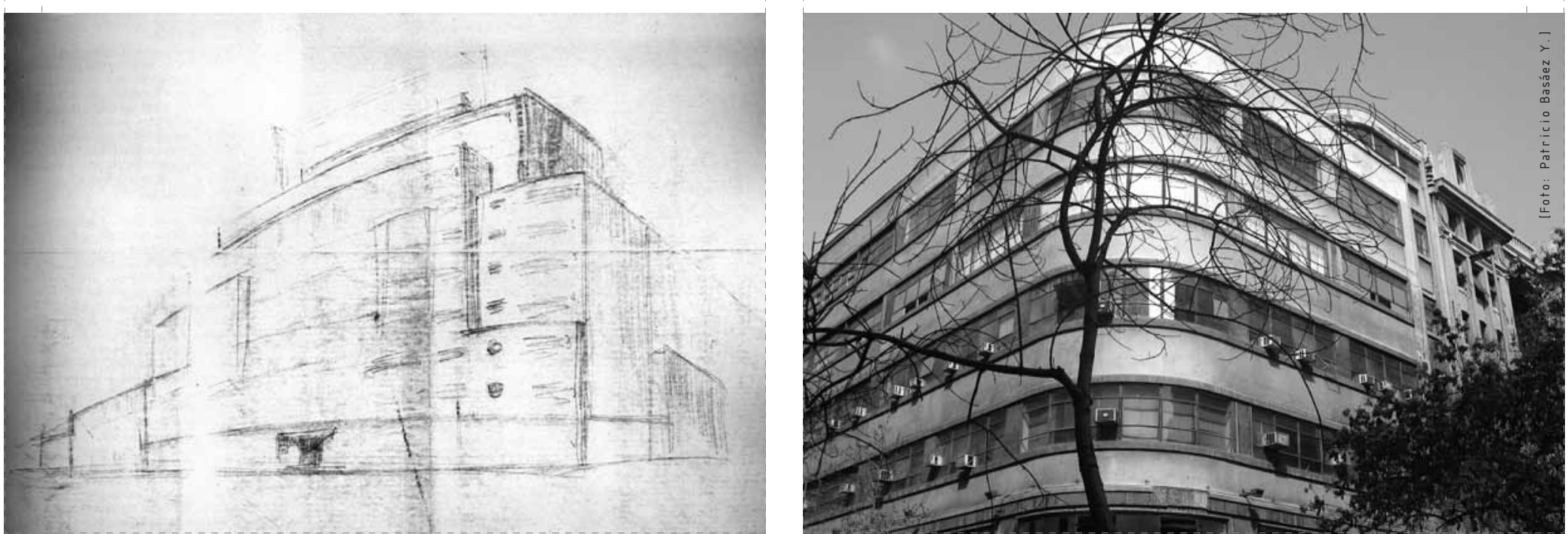

volar, me ensenó a despegar como arquitecto. Yo le decía «Jorge, yo aprecio mucho tu arquitectura y tu oficina y todo lo que se hace aquí, pero tú haces una arquitectura estereotipada y muerta, hay que hacer una arquitectura viva, yo quiero hacer arquitectura viva». Y un día Jorge me dijo: "Mira, el Banco Hipotecario nos ha entregado un edificio en la calle Estado esquina de Huérfanos, te lo doy a ti, proyéctalo tú. Yo voy estar contigo naturalmente, no te voy a dejar hacer tonteras, pero hazlo, proyéctalo tú». Y de ahí salió el Oberpaur.

Bueno, en ese mismo tiempo, tomé contacto con algunos grupos de arquitectos, que ya estaban en esta inquietud de la arquitectura moderna, de las dos universidades

(Universidad Católica y Universidad de Chile) Hicimos un grupo que se llamaba el Grupo Espacio, en que estaba Héctor Mardones ${ }^{27}$ estaba Ulriksen.

\section{¿Guillermo Ulriksen?}

-Sí, Guillermo Ulriksen ${ }^{28}$, estaba Alfredo Johnson, que hizo algunas cosas, estuvo estudiando urbanismo en Francia, llegó e hizo una casa en la calle Amunátegui, que la demolieron, fue una casa bien interesante.
¿El Oberpaur fue el primer edificio moderno que se hizo?

-Yo creo que sí.

\section{¿Y qué reacción produjo?}

-iUn espanto! A mí me insultaban en la calle, en serio, me insultaban en la calle. Que era un horror, que era vergüenza, me decían: «con la tradición que tú tienes, de haber tenido una buena educación, de haber estado en Europa, que traigas estas cosas espantosas aquí, de maquinismo», en fin, una reacción muy violenta. Es decir, tan violenta que la clientela de Arteaga, que era el Banco Hipotecario, la Chilena Consolidada, la Sociedad de Renta Urbana, es decir, las grandes empresas que construían, le dijeron a Arteaga: «mira, muy bien, sigue con Sergio Larraín, tenemos mucho aprecio por él, pero nada de Oberpaur, no seguimos con Oberpaur». Los otros edificios que seguimos haciendo ya no pudieron estar en esa línea, y se empezó poco a poco a crear entonces un distanciamiento entre Arteaga y yo a pesar que a mí me daba la posibilidad de hacer arquitectura. Hicimos edificios neoclásicos por ejemplo, como la Sociedad Nacional de Agricultura, que está al lado del Teatro Municipal. Es un edifico del cual no me arrepiento tampoco porque está a la escala de la plaza y está bien. Pero se hicieron otros edificios que a mí no me gustaban.
¿Qué eran clásicos también?

-Qué eran clásicos y eran góticos. Hacían lo que se les pedía y lo hacían muy bien. Lo hacían realmente con mucha seriedad. Eran gente que yo respetaba mucho por eso. Todo lo que se hacía en la oficina de él era de muy buena calidad. En varias oficinas se trabajaba así, con un buen nivel de calidad.

\section{¿Qué dice de Juan Martínez ${ }^{29}$ ?}

-Juan Martínez, en el fondo era un romántico. Yo me acuerdo que a Juan Martínez yo lo conocí a través de su obra un año antes de recibirme. En el año 27 hubo un congreso de arquitectura, de esos Congresos Panamericanos de Arquitectura en Buenos Aires, que fue cuando ocurrió lo de Alpatacal ${ }^{30}$, no sé si ustedes...

\section{¿La tragedia?}

- La tragedia en que murieron gran parte de los muchachos de la Escuela Militar chilena. Estábamos allá, creo que yo iba como presidente del Centro de Alumnos de la Facultad de Arquitectura de la Universidad Católica, y me parece que iba un muchacho de apellido Rivera de la Universidad de Chile. Llevaba como pieza de fondo para mostrar, por parte de la Universidad de Chile, acuarelas de Juan Martínez que nos dedicamos a colgar y

\footnotetext{
27 Héctor Mardones Restat (1907-1977), Arquitecto Universidad de Chile, Premio Nacional de Arquitectura 1973

8 Guillermo Ulriksen Becker Arquitecto Universidad de Chile.

29 Juan Martínez Gutiérrez (1901-1971). Arquitecto Universidad de Chile. Premio Nacional de Arquitectura 1969. Uno de los arquitectos chilenos más importantes del siglo xx autor de edificios emblemáticos como la Escuela de Derecho de la Universidad de Chile o el Templo Votivo de Maipú.

30 Siniestro ferroviario ocurrido en Mendoza en el año 1927
} 
que realmente eran muy lindas. Excelentes, muy buenas, muy, muy bonitas las acuarelas. Después vi el pabellón de Sevilla ${ }^{31} \ldots$

\section{¿El pabellón chileno para la exposición de Sevilla?}

-A mí me parecía que había una gran cantidad de cosas falsas. Sobre todo que el edificio es del año 1929, contemporáneo al Pabellón de Barcelona de Mies Van der Rohe. Entonces uno no entiende que puedan ser en España ambas cosas al mismo tiempo. Y fíjate que era casi contemporáneo al Oberpaur, con uno, dos años de diferencia y el Oberpaur en Chile que verdaderamente fue como un torpedo, me acuerdo también, que en ese tiempo hice una casa [en esa línea].

Dávila ¿estaba haciendo cosas modernas en esa época?

-Cuando yo llegué y comencé a trabajar, Roberto Dávila ${ }^{32}$ no estaba. Estaba creo que en Viena todavía. Dávila llegó después. Yo me acuerdo que lo invité a entrar en un concurso, y entramos juntos a un concurso.

\section{¿A cuál?}

-Un concurso que por supuesto no ganamos. Salimos seguramente en los últimos lugares. Era un concurso que hizo una persona que después fue Ministro de Hacienda aquí, Roberto Wachholtz ${ }^{33}$ quien abrió la Avenida Las Lilas con Avenida Providencia. Él tenía todo ese terreno y quería entonces que proyectáramos toda la entrada a esa avenida. Se hizo un concurso de anteproyectos, pero no se hizo ninguno, sino que vendieron los lotes, e hicieron un edificio de esquina. Pero con Dávila tuve muchas conversaciones y lo mismo con Rodulfo Oyarzún Phillipi ${ }^{34}$, bastante mayor que yo. Él estaba más bien metido en el Urbanismo, en temas más vinculados a la planificación que a la arquitectura.
¿Este Grupo Espacio era un ámbito de discusión?

-Mira en el fondo la verdad es que era una cosa bien superficial, pero fue como el primer intento de un grupo de gente que quería protestar.

\section{¿En qué año es eso?}

-Me es difícil decirte, pero debe haber durado unos dos o tres años, desde los años 29 ó 30 por ahí más o menos.

\section{¿Eran sólo arquitectos?}

-Sí, éramos todos arquitectos. Arrendábamos una casa que todavía existe, y que se ha conservado en una forma milagrosa, una casa chiquita muy pintoresca que está en el Parque Forestal casi frente a la Fuente Alemana. Esa casa era entre lugar de fiestas y sala de reuniones, colgábamos planos y sobre todo criticábamos. Lo que más hacíamos era criticar a todo el mundo porque realmente había una cosa más bien de rebeldía que de principios muy fuertes en nosotros.

\section{¿Quiénes estaban?}

-Me acuerdo de las personas que estaban de la Universidad de Chile, que eran más serias: eran Ulriksen, Mardones y de la Universidad Católica, me parece que los más serios también eran Alfredo Johnson, que incursionó un período en eso, y yo. Después había otras personas que yo no las he visto figurar después, que ni siquiera supe si habían ejercido la profesión.

\section{Ya eran todos titulados en esa época ¿no?}

-Mira, deben haber sido titulados o en los últimos años de la universidad.
Ahora respecto a esta trasposición del estilo moderno a Chile, ¿cómo ve usted que se hizo ese traspaso? ¿Fue una simple importación de moda o hubo realmente una motivación por un cambio ético, uno de contenido, una revolución de las formas sociales de agrupación, el problema de la vivienda masiva, o sea, si hubo realmente una preocupación de fondo, como la que sí había en Europa para producir ese cambio, esa industrialización?

- Te voy a contestar tal vez en una forma un poco elíptica. Yo tuve una mala experiencia de la arquitectura social en Europa. En el año 28 me recibí, hice muy rápida mi carrera, hice mi programa de estudios yo mismo, y lo solicité desde que entré a la escuela hasta que me recibí con mi título, me casé. Quería casarme lo más luego posible, ser arquitecto y salir de la escuela. Creo que lo hice en tres años y diez meses. Hice un programa y hacía proyectos de dos cursos diferentes y el otro año hacía los ramos de dos cursos diferentes, así que hice una cosa telescópica de toda la carrera y la apreté. Hice un programa que lo tuve que presentar a la facultad y me lo aprobaron. Yo les decía que lo natural era que hubiera diversas velocidades según el entusiasmo que tenían los alumnos para trabajar. Que era absurdo que el estudiante más lento fuera el que diera la pauta para el tiempo de todos los demás, que en ninguna parte se hacía eso, tal vez en los convoyes de guerra, pero en otras cosas no se hace. Bueno, y me lo aceptaron, entonces me recibí con Garáfulic-que estaba un año antes que yo- y nos recibimos juntos. También hizo el proyecto final en las vacaciones, desde diciembre, y yo me recibí en abril y me casé el 5 de mayo. Nos fuimos a Europa el 10 de mayo. Fui a la Bauhaus, que me interesaba mucho y que conocía por referencias, más bien por revistas.

Don Sergio, le agradezco su tiempo y su disposición. Muchas gracias.

31 Edificio proyectado por Juan Martínez Gutiérrez para representar a Chile en la Exposición Iberoamericana de Sevilla del año 1929.

32 Roberto Dávila Carson, Arquitecto Universidad de Chile. Obtiene el Premio Nacional de Arquitectura en el año 1971

33 Ministro de Hacienda de la República de Chile durante los años 1946 y 1947 , durante el gobierno de Gabriel González Videla.

34 Rodulfo Oyarzún Phillipi (1895-1985). Arquitecto Universidad de Chile, Urbanista, Pintor, Escultor, y Educador en las líneas de las Artes Plásticas, la Arquitectura y e Urbanismo. Premio Nacional de Arquitectura 1974. 